\title{
La racionalización de las infraestructuras de transporte en España*
}

\author{
Germà Bel \\ Universitat de Barcelona \\ $\mathrm{y}$ \\ Barcelona Graduate School of Economics
}

\begin{abstract}
Resumen
En las últimas décadas se han generalizado entre los países más desarrollados algunas prácticas de buen gobierno relativas a las inversiones en infraestructuras: la evaluación ex-ante de los proyectos, el establecimiento de Planes Económicos y Financieros, y la evaluación ex-post de los proyectos. Sin embargo, este tipo de prácticas características del buen gobierno no ha encontrado un eco signi ficativo en España. Este artículo muestra que las características del diseño institucional y organizati vo de los entes gestores de las grandes infraestructuras de transporte en España facilitan soslayar la evaluación de proyectos y el establecimiento de Planes Económicos y Financieros; de hecho, incenti van no hacerlos. El trabajo compara algunas reformas del diseño institucional y organizativo de las infraestructuras de transporte que permitirían avanzar más en los principios del buen gobierno en el ámbito de las infraestructuras de transporte.
\end{abstract}

Palabras clave: infraestructuras, transporte, evaluación

Clasificación JEL: H43, L92, R41, R42, R48.

\begin{abstract}
During the last decades, many developed countries are applying good governance practices to investments in infrastructure (e.g., long-term economic and financial project planning, both ex-ante and ex-post project evaluation). Unfortunately, these practices are not commonly used in Spain. This paper discusses how the institutional and organizational framework for large infrastructure projects in Spain does not promote the evaluation of projects, and neither the application of rigorous economic and financial planning: in fact, it provides negative incentives to do it. The paper proposes several ins titutional reforms to improve the organizational framework for transport infrastructure, which could help to apply good governance practices.
\end{abstract}

Keywords: infrastructure, transport, project evaluation.

JEL classification: $H 43, L 92, R 41, R 42, R 48$.

\section{Introducción}

La condición requerida para que una infraestructura contribuya al bienestar social ha sido expresada de forma clara y precisa en De Rus (2009): los beneficios sociales de la infraestructura han de ser mayores que los costes sociales que ha generado. El cumplimiento de esta condición exige tener en cuenta tres cuestiones que revisten importancia central al considerar la pertinencia de desarrollar un proyecto:

\footnotetext{
* Este trabajo ha disfrutado de la financiación del Ministerio de Ciencia y Tecnología (ECO200906946/ECON) y de la Generalitat de Catalunya (SGR2009-1066). El autor agradece las sugerencias de Daniel Albalate, Ángel de la Fuente, Ginés de Rus y Xavier Fageda.
} 
En primer lugar, ¿cuánto cuesta? Es decir, cuál es el monto de recursos requerido para la creación-o reforma- de la infraestructura, y para su operación posterior -durante su vida útil-. El concepto de coste se refiere sobre todo al coste financiero, pero no solo a éste. También es importante tener en cuenta, por ejemplo, los costes medioambientales implicados por la construcción de la infraestructura, aspecto habitualmente soslayado en la consideración del efecto medioambiental de proyectos.

En segundo lugar, ¿qué hace? Es decir, qué beneficios aportará la infraestructura tras su entrada en servicio. La mayor parte de estos beneficios proceden de los ahorros de tiempo de viaje facilitados por la nueva oferta, con el consiguiente aumento de la productividad de la economía. De hecho, es importante tener en cuenta que los beneficios de la nueva infraestructura serán determinados tanto por la atracción de tráfico de otros modos menos competitivos, como por la inducción de tráfico adicional. Además, es importante considerar las potenciales ganancias (o pérdidas) medioambientales que la operación de la nueva infraestructura hará posibles, así como otros potenciales efectos por los que la sociedad pueda tener preferencia, como la supresión o atenuación de situaciones de aislamiento territorial por lo que respecta a la movilidad.

En sustancia, las cuestiones ¿cuánto cuesta? y ¿qué hace? otorgan relevancia a la realización de análisis coste-beneficio. Además, todavía es necesario formular una última cuestión: ¿qué podría lograrse con un uso alternativo de los recursos exigidos por el proyecto en consideración? Es decir, cuál es el coste de oportunidad de los recursos requeridos por la infraestructura. Esto hace necesario comparar un proyecto concreto con proyectos alternativos, así como con la alternativa de no realizar acción alguna, que podría ser eventualmente la mejor opción.

En suma, una política de infraestructuras adecuada exige analizar la relación entre los costes y beneficios de los proyectos, así como el coste de oportunidad de los recursos requeridos ${ }^{1}$. Sin embargo, la política de infraestructuras en España, inmersa en una verdadera vorágine inversora durante la década de los 2000 , ha prescindido de una forma sistemática de la ayuda del análisis coste-beneficio riguroso y de la realización de planes económicos y financieros adecuados. Esto ha perjudicado gravemente la racionalidad económica y social de muchos de los proyectos acometidos.

Este tipo de práctica se ha visto favorecida por la extraordinaria afluencia de recursos de que han gozado las administraciones públicas españolas hasta la eclosión de la crisis económica a partir de 2008. También han podido influir factores de tipo cultural, como la existencia de una creencia casi incondicional en las bondades de prácticamente cualquier inversión en infraestructuras. Esta creencia fue generada por los efectos de la política inversora en décadas anteriores, las de los años 1980 y 1990, cuando España era todavía un país inmaduro en materia de infraestructuras.

${ }^{1}$ DE RUS (2009) ofrece una discusión detallada de las cuestiones implicadas por el análisis costebeneficio y por el análisis del coste de oportunidad. 
Sin embargo, tras un largo período de gran actividad inversora, España ya es un país maduro en cuanto a dotación de infraestructuras, por lo que la rentabilidad marginal de nuevas actuaciones tiende a ser mucho menor que en el pasado (Bel, 2007). De ahí que carezca de sentido el mantenimiento de tal adhesión incondicional a la idea de que toda actuación en infraestructuras es beneficiosa.

Además de esos factores de tipo fiscal y cultural, revisten también gran relevancia otros factores que se conectan con otra de las preocupaciones centrales de los economistas: los incentivos. Más en concreto, la existencia de incentivos derivados de las características institucionales y organizativas que gobiernan los diferentes modos de transporte y que fomentan la desconsideración del análisis coste-beneficio y del coste de oportunidad de los proyectos infraestructurales.

Este trabajo se ocupa especialmente de analizar y discutir la reforma de algunas características institucionales y organizativas de las grandes infraestructuras en España que contribuya a racionalizar la política de transporte. Estas reformas son necesarias, y también urgentes, dada la dimensión adquirida por los problemas existentes. De hecho, el mes de octubre de 2010, en el que este trabajo fue acabado de redactar, nos ha ofrecido tres buenos ejemplos de esto, en diferentes modos de transporte. Una breve revisión de estos casos permite observar la entidad del problema. Con este propósito, el trabajo se estructura de la siguiente forma: para cada tipo de infraestructura considerado (aeropuertos, ferrocarriles y carreteras) ${ }^{2}$ se presenta, en primer lugar, un caso ilustrativo de práctica deficiente. A continuación se discute cómo el diseño institucional y organizativo de la política en el correspondiente modo ha facilitado este tipo de prácticas, y qué tipo de reformas contribuirían a racionalizar las decisiones de inversión y de gestión en el modo. Finalmente, se discuten las principales implicaciones de política pública derivadas del análisis.

\section{Inversión y gestión en los aeropuertos}

\subsection{La ampliación del aeropuerto de León}

El 11 de octubre de 2010 fue inaugurada la nueva terminal del aeropuerto de León. El coste de la terminal ha superado los 29 millones de euros. La terminal propiamente dicha ha supuesto una inversión de 22,1 millones de euros, mientras que a

\footnotetext{
${ }^{2}$ Los puertos presentan unos problemas similares a los de los aeropuertos, por lo que las consideraciones efectuadas para estos últimos son aplicables a aquellos por analogía. No obstante, es bueno notar que la dimensión de los problemas es menos en el caso de los puertos por, entre otros, dos motivos importantes. Por un lado, porque los puertos se dedican fundamentalmente al transporte de mercancías, y -desafortunadamente- éste ha ejercido menor magnetismo sobre los decisores políticos que el transporte de viajeros. Por otra, porque existe un cierto grado de individualización de la gestión de los puertos en España que, aunque moderado, ha favorecido una mayor eficiencia en las decisiones sobre inversiones y sobre la operación de los puertos.
} 
la renovación de la central eléctrica se han dedicado 6,9 millones de euros ${ }^{3}$. Además, esta inversión había sido precedida por otras actuaciones, entre la que destaca la ampliación de la pista de aterrizaje y estacionamiento de aeronaves, cuyo coste ha sido de 42 millones de euros. En total, y considerando otras inversiones menores, la inversión total efectuada se acerca a 80 millones de euros en los últimos seis años. Como consecuencia de la ampliación la capacidad de asistencia pasajeros/hora ha pasado de 180 a 580 usuarios, multiplicándose por tanto por 3,2. El volumen de pasajeros que puede gestionar la nueva instalación se sitúa bien por encima de un millón al año, y con ello se pretende, según declararon las autoridades aeroportuarias en la inauguración, «multiplicar el 'magnetismo' [sic] económico y social de León». 4

Sin ningún género de dudas, la ampliación de la capacidad del aeropuerto permite absorber un gran aumento de demanda en el futuro. Sobre todo si tenemos en cuenta que los pasajeros gestionados por el aeropuerto de León en los últimos años han sido muchos menos ${ }^{5}$. El récord de pasajeros se alcanzó en 2007, con 161.705. No obstante, en 2009 gestionó sólo 95.128 pasajeros, y el acumulado en los nueve primeros meses de 2010 ha sido de 72.422 pasajeros, con un descenso interanual del 4,8 por 100 hasta septiembre. Por tanto, la nueva terminal puede absorber un tráfico que es más de 10 veces superior al actual. Aunque deberá hacerlo en unas circunstancias adversas. Esto es así no solo por la eventualidad de un período largo de crecimiento económico muy moderado. Además, se da la circunstancia de que, como anunció el gobierno en el mismo acto de inauguración de la nueva terminal del aeropuerto, en 2012 está prevista la puesta en servicio de la conexión de alta velocidad ferroviaria entre León y Madrid, con un tiempo de viaje de unas dos horas, que previsiblemente afectará gravemente a los vuelos con destino a la capital, que es uno de los dos únicos servicios non-stop con frecuencia diaria (además de Barcelona) servidos desde León. Sólo cabe añadir que no se conoce análisis coste-beneficio de la ampliación del aeropuerto de León, y que no es necesaria en sentido estricto la elaboración de un Plan Económico Financiero, pues el aeropuerto se administra financieramente con el sistema de caja única de AENA.

\footnotetext{
${ }^{3}$ Las principales características de la inversión realizada en el aeropuerto de León pueden encontrarse en la nota de prensa elaborada por el Ministerio de Fomento con motivo de la inauguración de la ampliación, y que está disponible en el enlace http://www.fomento.es/NR/rdonlyres/36704FF0-984941A0-B992-E5D36909E63D/77936/10101103.pdf (descargado el 31 de octubre de 2010).

4 Véase http://www.europapress.es/economialtransportes-00343/noticia-economia-nueva-termi nal-aeropuerto-leon-casi-triplicara-capacidad-tasas-reduciran-22-20101011212511.html (descargado el 31 de octubre de 2010)

${ }^{5}$ La carga de mercancías gestionada es muy escasa. Los datos de tráfico pueden encontrarse en la página web de AENA (http://www.aena.es).
} 


\subsection{Modelo de gestión de los aeropuertos en España: de la gestión conjunta a la individual}

La empresa pública Aeropuertos Españoles y Navegación Aérea (AENA), adscrita al Ministerio de Fomento, gestiona de forma conjunta prácticamente todos los aeropuertos que canalizan tráfico comercial en el territorio español. Además de gestionar las instalaciones aeroportuarias, AENA se encarga también de gestionar el control del tráfico aéreo, ámbito éste que escapa del objeto de nuestro análisis ${ }^{6}$. AENA es la propietaria de todas las instalaciones asociadas a los aeropuertos, y juntamente con el Ministerio de Fomento juegan un papel decisivo en todos los aspectos centrales de la actividad aeroportuaria, como pueden ser las decisiones sobre inversiones, la asignación o coordinación -según el caso- de los derechos de aterrizaje y despegue (slots), o las negociaciones que se establecen con las compañías aéreas. España constituye un caso excepcional porque es el único país occidental con varias ciudades y aeropuertos de grandes dimensiones donde los aeropuertos son gestionados exclusivamente por una única entidad dependiente del gobierno central. Sólo el caso de Rumania es similar, aunque el tamaño del mercado aéreo en este país es muy inferior ${ }^{7}$.

Dadas las características de la gestión de los aeropuertos en España, la asignación de los recursos económicos generados por la actividad aeroportuaria se realiza a través del mecanismo de la caja única. La caja única supone la existencia de un sistema de subvenciones cruzadas entre los aeropuertos españoles, que no es transparente ni eficiente. Por otra parte, las tasas cobradas a las compañías aéreas por el uso de las instalaciones aeroportuarias son aprobadas por las Cortes Generales, y se establecen para tres categorías de aeropuertos a partir de los niveles de tráfico alcanzados, de forma que (1) la diferenciación entre las tres categorías de aeropuertos es arbitraria, y no responde a consideraciones de coste, y (2) no existe la posibilidad de diferenciación de precios entre aeropuertos de una misma categoría. La clasificación en tres categorías a efectos de percepción de tasas fue establecida en 1957, y se ha mantenido vigente hasta la actualidad, con algunos cambios en la composición de cada categoría. A partir de 2011 los dos mayores aeropuertos, Madrid-Barajas y Barcelona-El Prat serán singularizados a efectos de fijación de tasas, de acuerdo con las previsiones del proyecto de Ley de Presupuestos Generales del Estado para 2011.

Estos factores que distorsionan la fijación de precios y la competencia tienen un efecto que puede ser muy perjudicial sobre la eficiencia. La política de fijación de tasas aeroportuarias disocia la decisión sobre tasas de cada aeropuerto de la financiación de los costes derivados de la inversión efectuada en el aeropuerto en cues-

\footnotetext{
${ }^{6}$ En BEL y FAGEDA (2011) puede encontrarse información actualizada sobre los modelos de gestión aeroportuaria en Europa.

7 Véase http://www.europapress.es/economia/transportes-00343/noticia-economia-nueva-termi nal-aeropuerto-leon-casi-triplicara-capacidad-tasas-reduciran-22-20101011212511.html (descargado el 31 de octubre de 2010).
} 
tión, y de los gastos derivados de su operación. Además, no ofrece señales correctas respecto a la inversión futura, pues la demanda es sobrevalorada a causa de los precios bajos. Por su propio diseño, el modelo de gestión promueve la ineficiencia de las inversiones, puesto que estas no necesitan estar relacionadas con la capacidad de generación de ingresos de cada aeropuerto, que depende crucialmente de la demanda de servicios aéreos.

La ineficiencia en las inversiones efectuadas así como la inadecuación de las tasas percibidas por el uso de las instalaciones puede contribuir a explicar que el umbral de tráfico para el que los aeropuertos españoles tienen autosuficiencia financiera se encuentre muy por encima de los umbrales hallados en estudios realizados para la Comisión Europea comparando diferentes países ${ }^{8}$. De acuerdo con estos estudios para la CE, los aeropuertos con más de un millón de Unidades de Carga $(\mathrm{UdC})^{9}$ tienden a ser autosuficientes (depreciación incluida) y «los que no lo hacen, o bien están ofreciendo grandes descuentos en sus tasas aeronáuticas, o bien tienen algunos problemas fundamentales de eficiencia en su gestión» (European Commission, 2002, págs. 5-33). En el caso de España, sin embargo, todos los aeropuertos con menos de tres millones de UdC, excepto el de Murcia-San Javier, experimentaron resultados negativos en 2009, y solo a partir de cinco millones de UdC se encuentran resultados brutos de operación sistemáticamente positivos (Bel, 2010, págs. 224-227). No obstante, los dos principales aeropuertos, Madrid y Barcelona, registraron también resultados negativos, dado que las grandes inversiones efectuadas en ambos no pueden financiarse con las tasas que se aplican por el uso de las instalaciones.

El tipo de reforma necesario para introducir mayores incentivos a la eficiencia tanto en las decisiones de inversión como en la gestión técnica es clara: el cambio al modelo de gestión individual, siguiendo la pauta común en los países desarrollados de tamaño y mercado aéreo comparable al español. La gestión individual ofrece mayores incentivos a alinear las decisiones de inversión con el potencial de generación de ingresos del aeropuerto. Qué administraciones hayan de tener un carácter preeminente en la gestión del aeropuerto y qué fórmula de gestión específica en la dimensión público-privado se adopte son cuestiones secundarias en este sentido. Ciertamente, la individualización de la gestión de aeropuertos no elimina la posibilidad de que se realicen inversiones carentes de sentido económico y social, como muestran los aeropuertos de Ciudad Real y de Lleida ${ }^{10}$, que han entrado en servicio en los últimos años. No obstante, las mayores dificultades para la financiación de déficits sistemáticos a escala subcentral limitan las posibilidades de que estas prácticas se extiendan en la misma medida. Además, la disciplina que la gestión indivi-

\footnotetext{
${ }^{8}$ La eficiencia técnica en la gestión de los aeropuertos de AENA es manifiestamente mejorable. Este aspecto ha sido ilustrado empíricamente en trabajo como MARTÍN, ROMÁN y VOLTES-DORTAS (2009), BEL (2010) y BEL y FAGEDA (2011).

${ }^{9}$ Unidad básica de volumen de tráfico. Un pasajero es equivalente a $100 \mathrm{~kg}$. de carga. Por tanto, el total de unidades de carga proceden de sumar 'número de pasajeros' + kilos de mercancías/100.

${ }^{10}$ En el caso de Lleida, aeropuerto de competencia autonómica, puede considerarse que existe un modelo de gestión conjunta a nivel autonómico, similar al de AENA aunque a escala regional.
} 
dual impone a todos los aeropuertos puede limitar la magnitud de las subvenciones necesarias para sostener a los deficitarios (Mills, 1995).

No sería realista discutir la reforma del modelo de gestión de aeropuertos en España sin considerar el asunto de la solidaridad, que ha sido el gran argumento que se ha usado tradicionalmente para justificar el mantenimiento del modelo de gestión conjunta. En este sentido, sería deseable que se discutiese de forma abierta y precisa cuales son aquellos aeropuertos que cumplen una obligación de servicio público (OSP), en el sentido de que su existencia evita el aislamiento y facilita la movilidad -en términos modernos- en territorios en que la densidad de demanda no sea suficiente para que un aeropuerto pueda ser autosuficiente. En el caso de España, esta situación se limita probablemente a la de algunas islas menores. En estos casos sería deseable establecer mecanismos de subvenciones a los aeropuertos deficitarios, que en un sistema individual se pueden abordar de diferentes formas. Por un lado, se puede realizar mediante subvenciones específicas a los aeropuertos. Tales subvenciones, sean de capital o corrientes, pueden proceder del gobierno central (como en Irlanda), del gobierno regional (como en Alemania), de los gobiernos locales (como en Australia y Reino Unido), o de todas las Administraciones (como en Italia).

Por otro lado, la financiación de los aeropuertos deficitarios puede llevarse a cabo también mediante un fondo creado con parte de los excedentes generados por los grandes aeropuertos, como ilustra el caso de Canadá. Las subvenciones pueden aplicarse también a las compañías aéreas, como fórmulas alternativas de sostener a los aeropuertos deficitarios en la medida que se garantiza un nivel mínimo de tráfico (William y Pagliari, 2004; Nolan, Ritchie y Rowcroft, 2005). Los casos de Irlanda y Reino Unido muestran la importancia que pueden llegar a tener estos mecanismos de apoyo a las compañías aéreas. También pueden imponerse obligaciones de servicio público a las compañías aéreas en el marco de la normativa europea, que establezcan una regulación de entrada, de precios y de frecuencias (como se hace ya en rutas intra-insulares en Canarias y en Baleares).

En cualquier caso, lo que está muy claro es que no es necesario tener un modelo centralizado como el español para articular mecanismos de apoyo financiero a los aeropuertos con déficit y dificultades para la autofinanciación que -no obstante- se considere necesario mantener en operación. Además, los mecanismos de ayudas en modelos de gestión individual, por su transparencia, evitan situaciones como la que se registra en España, donde los aeropuertos que reciben mayor subvención de la caja común son los dos mayores, Barcelona y, sobre todo, Madrid (Bel y Fageda, 2011).

\section{Inversión en la red de alta velocidad en España}

\subsection{La entrada en servicio de la línea de Alta Velocidad Madrid-Valencia.}

El 13 de octubre de 2010 se efectuó el viaje oficial de prueba del tren de alta velocidad que a partir de diciembre del mismo año conecta regularmente las ciuda- 
des de Madrid y Valencia, sirviendo también las ciudades de Cuenca y Albacete. De acuerdo con las cifras disponibles en la Memoria Económica para el ejercicio 2009 de Adif, la inversión contratada a 31 de diciembre de 2009 para la línea MadridValencia era de 6.629 millones de euros, y la longitud de la nueva infraestructura de 364,7 kilómetros (a los que hay que añadir los 28 kilómetros ya en servicio en el corredor Madrid-Sevilla, con los que conecta la línea Madrid-Valencia). Tras la entrada en servicio de esta nueva línea, la red de AVE cuenta con 1.964 kilómetros en servicio (a velocidad $>250 \mathrm{~km} / \mathrm{h}$ ), y es la más extensa de la OCDE, y segunda del mundo, tras la de China. Así pues, la red de AVE es más extensa que la de los dos países pioneros del AVE: Japón, que cuenta con 1.875 km. y Francia, con 1.872. Esto a pesar de que los viajeros transportados por Alta Velocidad en España son poco más del 5 por 100 de los transportados por el Shinkansen en Japón y poco más del 15 por 100 de los transportados por el Train à Grande Vitesse (TGV) en Francia.

La nueva línea Madrid-Valencia, al igual que las entradas en servicio con anterioridad, no fue sometida antes de su aprobación a un análisis coste-beneficio propiamente dicho ${ }^{11}$. No obstante, a raíz del viaje de prueba del 13 de octubre, las autoridades del Ministerio de Fomento dieron a conocer los resultados de un análisis del impacto económico de la nueva línea, aunque no se trata de un análisis coste-beneficio en sentido estricto, pues no se tienen en cuenta los costes. Los principales elementos de la información proporcionada son los siguientes ${ }^{12}$ :

- La demanda estimada para la línea es de 3,6 millones de viajes en los primeros años, de los cuales 2,8 millones serán captados de otros modos (la mayor parte del punto a punto entre Madrid y Valencia), y 800.000 viajes serán tráfico inducido. El AVE captará el 55 por 100 del tráfico aéreo, el 25 por 100 del coche y el 5 por 100 del autobús. En conjunto, la atracción de tráfico ya existente en otros modos es mucho más importante que la inducción de tráficos nuevos.

- Según el estudio económico presentado, el AVE aportará cuantiosos beneficios económicos gracias sobre todo «al ahorro de 21 millones de horas anuales de desplazamientos» [sic], cuyo valor económico es de 408,5 millones de euros

${ }^{11}$ No obstante, están disponibles en la literatura económica evaluaciones coste-beneficio realizadas desde el ámbito académico para la línea Madrid-Sevilla (DE RUS e INGLADA, 1993, 1997) y la Madrid-Barcelona (DE RUS y ROMÁN, 2006).

12 La información dada a conocer está disponible en sendos documentos accesibles en la página web del Ministerio de Fomento. Por una parte, la nota de prensa elaborada con motivo del viaje http://www.fomento.es/NR/rdonlyres/A1E3D697-A480-4A97-BB5E-3E883C3D5B35/ 77973/10101301.pdf (descargada en 31 de octubre de 2010). Por otra, la presentación del estudio El impacto económico de la línea de alta velocidad Madrid-Valencia, elaborada por el centro de Estudios Económicos Tomillo, de la Fundación Tomillo, cuya actividad está centrada en programas de intervención social (http://www.tomillo.org/v_portal/apartados/apartado.asp). La presentación está disponible en la página web del Ministerio de Fomento ( http://www.fomento.es/NR/rdonlyres/A1E3D697A480-4A97-BB5E-3E883C3D5B35/77981/101013PresentacionestudioAVEValencia.pdf, descargada el 31 de octubre de 2010). 
(pág. 17 de la presentación), y al gasto de 865 millones de euros anuales por los viajeros del AVE. En conclusión el pronóstico es apabullante: el AVE generará 136.000 empleos entre 2010-2016.

- Los estudios realizados indican que el uso del AVE permitirá una ahorro de 842.000 toneladas de emisiones de $\mathrm{CO}_{2}$ hasta 2016 , lo que implica un promedio de unas 140.000 toneladas anuales de emisiones de de $\mathrm{CO}_{2}$ evitadas.

Claro es que los cálculos en los documentos mencionados presentan algunos problemas no menores. En primer lugar, dado que la atracción de otros modos es 2,8 millones de pasajeros, para ahorrar 21 millones de horas al año, el viaje actual debería estar en 7,5 horas de promedio. Teniendo en cuenta que el ahorro respecto al avión (puerta a puerta) es inferior a una hora, y de unas dos en el caso del coche, al igual que en el caso del tren convencional, y que estos son los principales modos de origen... el AVE necesitaría captar algunos cientos de miles de viajeros procedentes de medios realmente decimonónicos para generar tamaño ahorro anual de horas ${ }^{13}$.

Por otra parte, puede observarse con los propios datos aportados por el ministerio que, dado que generalmente los viajes son de ida y vuelta, los aproximadamente 1,8 millones de viajeros (suponiendo que todos viajen punto a punto MadridValencia, y todos lo hagan con destino a Valencia) gastarán un promedio de 481 euros. No está mal, aunque hay un pequeño problema: 1,4 millones de viajeros ya viajaban antes, y probablemente gastaban más, pues el AVE disminuye el número de pernoctaciones por viajero. Por tanto, los 400.000 viajeros nuevos deberán gastar más de 2.162 euros cada uno en Valencia (recuérdese que estamos obligados a suponer que todos viajan a Valencia, aunque en la vida real el nodo secundario acostumbra a ser más frecuentemente origen que destino) ${ }^{14}$. Se intuye un gasto ciertamente elevado en unos viajes que son muy mayoritariamente de negocios y muy cortos, sobre todo en tiempos de escasas alegrías económicas, que afectan fuertemente a los gastos de representación.

Por último, existe un detalle a tener en cuenta por lo que respecta al beneficio medioambiental del AVE. Si consideramos que el precio de los derechos de emisión de de $\mathrm{CO}_{2}$ en septiembre de 2010 era de 14 euros por tonelada, el valor económico

\footnotetext{
${ }^{13}$ Esto incluso si se toma en cuenta, como en el estudio presentado, que las horas de AVE también son horas ahorradas, dada la disponibilidad de teléfono móvil y de internet. Esta extravagante forma de razonar (pág. 17 de la presentación) sólo permitiría incrementar en poco más de tres millones adicionales las horas liberadas 'para el trabajo productivo'. Esto dando por supuesto que el usuario tipo trabaja productivamente durante todo su viaje en AVE, pero no puede hacer absolutamente nada que sea productivo en ninguno de los medios alternativos.

${ }^{14}$ El Ministerio de Fomento no debe creer en realidad que todos los viajeros tendrán destino Valencia, pues el 19 de octubre presentaba un estudio en Madrid, en este caso realizado por Accenture, sobre el AVE Madrid-Levante, cuyo relación principal es la Madrid-Valencia, que sugería la creación de 85.000 puestos de trabajo en la Comunidad de Madrid. Seguramente los supuestos en este estudio eran diferentes, y la mayor parte de los pasajeros tenían como destino Madrid. Las notas de prensa y el estudio están accesibles en http://www.fomento.es/NR/rdonlyres/65E4292D-FFAA-47AA-9C65AB65E09E4AFC/78161/10101901.pdf (descargado 1 de noviembre de 2010)
} 
de las emisiones efectuadas es ¡de menos de 2 millones de euros al año! Parece poca eficiencia medioambiental, dado el ingente volumen de recursos empleados en la inversión. Y las cosas empeorarían si se hubiese considerado como coste las emisiones de $\mathrm{CO}_{2}$ provocadas por la actividad constructiva durante la ejecución de las obras de infraestructura (Kageson, 2009).

En suma, el AVE Madrid-Valencia debe añadir a sus costes de construcción el incurrido por los estudios de impacto económico encargados, que no parecen haber generado un beneficio -económico- estimable, aunque sí han generado fuerte impacto comunicativo en la prensa regional valenciana.

\subsection{Reconsideración de las inversiones en Alta Velocidad ferroviaria en España}

La inversión para la extensión de la alta velocidad ha sido muy elevada En conjunto, la inversión acumulada en el AVE hasta 2010 se acerca a 45.000 millones de euros nominales (incluyendo líneas en servicio y líneas en construcción) ${ }^{15}$. El volumen de inversión empleado es impresionante, pero el tráfico total es muy escaso. Esto sugiere que las tasas de retorno de la AV en España son paupérrimas ${ }^{16}$. La principal razón es clara: el volumen de población servida es pequeño y la competitividad del avión es mayor en el tráfico doméstico que en otros países, dada la geografía y estructura urbana de España. De hecho, las ganancias de tiempo (ahorro) son escasas respecto al avión. Este problema solo hará que agravarse con la entrada en servicio de nuevas líneas de AVE en el futuro, pues la planificación ferroviaria -incluida en el Plan Estratégico de Infraestructuras de Transporte (PEIT) 20052020 - contempla la conexión de todas las capitales de provincia con Madrid.

La responsabilidad de las inversiones en infraestructura de alta velocidad recae sobre la administración general del Estado, que la ejerce a través de Administrador de Infraestructuras Ferroviarias (Adif), entidad pública empresarial dependiente del Ministerio de Fomento. Los objetivos declarados expresamente por Adif son hacer del ferrocarril el medio de transporte por excelencia y facilitar el acceso a la infraestructura en condiciones de igualdad (www.adif.es). Estos objetivos son difíciles de lograr; especialmente el segundo, pues es materialmente imposible que todos los ciudadanos de España tengan «acceso a la infraestructura en condiciones de igualdad». La traducción práctica de este principio ha sido considerar como elemento

\footnotetext{
${ }^{15}$ En ALBALATE y BEL (2011) se pueden encontrar detalles concretos sobre las inversiones efectuadas en la extensión de la red de AVE desde la primera línea construida, la Madrid-Sevilla, que entró en servicio en 1992.

${ }^{16}$ El desarrollo del AVE se ha hecho sin realizar análisis coste-beneficio en sentido estricto, aunque la literatura académica ofrece algunos análisis de este tipo. Por ejemplo, de RUS e INGLADA (1993) mostraron que la línea Madrid-Sevilla no tenía ninguna justificación económica, por su saldo social negativo, y obtenían una Tasa Interna de Rendimiento del 0,52 por 100 para la línea. Incluso los análisis realizados para la línea Madrid-Barcelona muestran que la demanda no compensa la fuerte inversión realizada, pues los ahorros de tiempo son poco importantes (DE RUS y ROMÁN, 2006).
} 
constitutivo de igualdad que todos los ciudadanos (peninsulares) estén conectados en alta velocidad con la capital política de España, y tomando la capital provincial como unidad de medida del acceso igualitario. Esto explica la impresionante extensión de la red efectuada en España que es ya, como se ha mencionado, la segunda más larga del mundo para velocidades superiores a $250 \mathrm{~km}$ por hora.

Como es lógico, muy escasa racionalidad económica podía esperarse de unos objetivos que nunca han tenido la racionalidad económica (generar más beneficios sociales que costes sociales) como factor a tener en cuenta. En este caso, a diferencia del de los aeropuertos, no cabe plantearse la individualización organizativa de la gestión, puesto que estamos en presencia de una infraestructura de red caracterizada por el viaje a media y larga distancia. Por tanto, es necesario definir condiciones alternativas de reforma. Algunas condiciones podrían ser de gran utilidad. En primer lugar, la obligatoriedad de lograr una tasas de rentabilidad social por encima de algún umbral mínimo definido con carácter general, que exprese el coste de oportunidad de los recursos. Algunas referencias del entorno serían una tasa social de descuento superior al 8 por 100 -como se usa en Francia y en Reino Unido- o al 10 por 100 -como se usa en Estados Unidos y en Canadá- (Robusté, 2010). En segundo lugar, y conectando con la condición previa, ausencia de subvenciones presupuestarias a la ejecución de los proyectos de alta velocidad ferroviaria. El transporte ferroviario de pasajeros a larga distancia no tiene la consideración de obligación de servicio público en la Unión Europea, y sus externalidades (en aquellos casos en que son positivas) son tan extraordinariamente limitadas que no justifican ayudas relevantes. En tercer lugar, en caso de que se mantengan las subvenciones a la inversión en infraestructura, la exigencia de cofinanciación por las administraciones regionales interesadas en el proyecto limitaría adicionalmente el problema de una extensión tan irracional de la red como la seguida en España.

La sujeción de nuevas actuaciones a condiciones de este tipo no provocaría menoscabo alguno a la solidaridad interterritorial, si esta es una preocupación de los poderes públicos que esté reflejando preferencias colectivas. De hecho, la alta velocidad ferroviaria ejerce un efecto succión muy importante, que implica que el nudo más relevante de la red absorbe actividad económica de los puntos medianos y menores. De ahí que el efecto de la limitación de la extensión de la red sería muy probablemente positivo desde el punto de vista del reequilibrio territorial. Un problema de mayor entidad puede presentarse con el desequilibrio entre la sobreinversión realizada en las regiones en que la red se ha extendido ya de forma significativa con respecto a aquellas regiones en las que esto todavía no se ha producido. En este sentido, podrían adoptarse políticas compensatorias que prioricen las inversiones en regiones 'infrainvertidas', aunque tal inversión debe concentrarse en los proyectos con mayor rentabilidad social en la región, que probablemente correspondan a modos distinto al ferroviario, o a medios distintos a la alta velocidad dentro del modo ferroviario, como la modernización del ferrocarril de mercancías. 


\section{Inversiones y financiación de la red de carreteras de alta capacidad}

\subsection{El 'rescate' progresivo de las autopistas radiales de acceso a Madrid.}

El 26 de octubre de 2010 trascendió públicamente que una enmienda del Grupo Parlamentario Socialista al proyecto de Ley de Presupuestos Generales del Estado para 2011 eleva hasta 250 millones de euros los 135 inicialmente previstos para créditos participativos de tres radiales de Madrid, la R-2 (Autopista del Henares, alternativa a la A-2), la R-3 (alternativa a la A-3) y la R-5 (alternativa a la A-5). Los créditos participativos afectan también a la Ausur (Alicante-Cartagena). Estos 250 millones se añadirán a los 200 millones aprobados en el presupuesto para 2010, todo ello dirigido a compensar a las concesionarias por el elevadísimo sobre coste de las expropiaciones ${ }^{17}$.

Todas estas autopistas fueron aprobadas en la primera fase del Programa de Autopistas de Peaje de 1997 del gobierno central, presentada en febrero de 1997, y, junto a la R-4, se consideraron en ese momento como las nuevas concesiones que presentaban perspectivas de rentabilidad financiera. Las autopistas radiales de acceso a Madrid comenzaron a entrar en servicio a partir de 2004. Efectivamente, el coste de las expropiaciones ascendió muy por encima de lo previsto, a causa de diversas resoluciones judiciales. Pero, además de esto, la demanda de estas autopistas es mucho más baja de lo estimado al otorgar las concesiones, situándose en el entorno de los 10.000 a 15.000 vehículos día. Se da la circunstancia de que las autopistas libres alternativas a estas radiales han sido objeto de mejora en los últimos años por parte de la misma administración concedente. El caso más espectacular es el de la autopista de peaje Madrid-Toledo, cuya Intensidad Media Diaria en 2009 fue de 2.364 vehículos (aunque la previsión inicial era de 25.000), y se da la circunstancia de que tiene una autopista libre autonómica alternativa que no estaba prevista en el momento de efectuarse la concesión.

El bucle en el que se encuentra inmerso el gobierno central en esta materia es notable: en caso de quiebra de estas concesiones el Estado debería asumir entre 4.000 y 5.000 millones de euros de deuda de las concesiones que eventualmente quebrasen. El importe total de ayudas que el Estado debe ofrecer para evitar la quiebra de las concesiones de las autopistas concesionadas a finales de los años 1990 ha sido estimado por el gobierno en un total de 1.400 millones de euros ${ }^{18}$. Hay que tener en cuenta, no obstante, que para evaluar la conveniencia de elegir entre el subsidio público (1.400 millones) y la asunción de la deuda por quiebra de las concesiones (entre 4.000 y 5.000 millones) hay que considerar la generación de futuros

\footnotetext{
${ }^{17}$ Información detallada puede encontrarse en la página 8, del suplemento Negocios, de El País de 31 de octubre de 2010.

${ }^{18}$ Según declaración del Ministro de Fomento el 10 de noviembre de 2010 (http://www.expan sion.com/2010/11/10/empresas/1289384435.html? $a=$ OR2661404fd7ccd0a0df330f1985719b8b6\& $t=1$ 289440264), descargado el 10/11/2010.
} 
ingresos por peaje (incluida la posibilidad que ofrece la gestión conjunta de todas las vías de gran capacidad) que el Estado obtendría en caso de pasar a ser el gestor de las concesiones y, por tanto, receptor de estos ingresos. En todo caso, el dilema de política pública creado es una de las consecuencias de haber otorgado concesiones para autopistas de peaje que iban a contar con autopistas libres alternativas desde el inicio, objeto además de mejora en los últimos años.

\subsection{Racionalización de la inversión y el modelo de financiación de las autopistas en España}

El análisis económico de la cooperación público privada en infraestructuras de transporte sugiere que el uso de los partenariados público privados (PPP), puede ser un elemento importante para disminuir el riesgo de la realización de «elefantes blan$\cos { }^{19}$. Un requisito necesario para que esto se produzca es que la financiación de la infraestructura se produzca mediante el pago por el usuario, porque se asume que el sector privado no estará interesado en participar en proyectos cuyas expectativas de financiación son dudosas. Pero este requisito no es suficiente, como muestra el caso de las autopistas concesionadas al sector privado a finales de los años 1990 en España. Es necesario que los contratos de concesión realicen una correcta asignación de riesgos entre el gobierno y el inversor privado.

Por otra parte, la situación puede complicarse aún más por un motivo no menor: algunas inversiones pueden ser demandadas por grupos privados como fórmula para realizar inversiones financieras con rentabilidad garantizada. Este es un supuesto de ocurrencia bastante frecuente en España, dada la existencia de un sector empresarial en el sector de la construcción con gran peso económico y gran capacidad de influencia política. De ahí que los PPP, que pueden tener algunas ventajas en determinadas situaciones y con determinadas condiciones, no constituyan una garantía contra la ejecución de «elefantes blancos». Especialmente cuando existe, como es el caso de España, el 'sobreentendido' de que el gobierno evitará la quiebra de concesiones tanto por el coste político de tal evento como por el efecto disuasorio sobre las posibilidades de recurso al sector privado en futuros proyectos, que es visto como instrumento de reserva por los gobiernos en momentos de crisis presupuestarias.

El hecho concreto es que España es el segundo país de Europa en cuanto a densidad relativa de dotación de autopistas, solo detrás de Portugal. Es muy escaso el número de tramos de carretera con una intensidad de circulación actual -o en el futuro próximo- que haga conveniente aumentar la calidad de servicio mediante el aumento de capacidad -por reforma de la vía existente o por creación de nueva infraestructura.

\footnotetext{
19 Se denomina como «elefantes blancos» a los proyectos de inversión con rentabilidad social negativa (ROBINSON y TORVIK, 2005).
} 
Hace tiempo que es necesario repensar la forma en que se gestiona la red de vías de gran capacidad. Cada vez más las necesidades de la red son de mantenimiento y reposición, y esto crecerá aún más en el futuro. Esto en un contexto en que la red de autopistas en España (es decir, autopistas de peaje y autopistas libres de peaje -también denominadas autovías en España-) presenta unas características híbridas muy marcadas: Los peajes cubren aproximadamente el 25 por 100 de la red de autopistas a cargo del Estado, todas ellas sometidas a explotación mediante concesión a empresas privadas. El resto de autopistas son libres de peaje.

El caso del sistema mixto de España es singular. Entre los países de la UE, algunos tienen un modelo de financiación de autopistas fundamentalmente basado en los peajes, que cubren casi toda la red de autopistas y están distribuidos regularmente a lo largo de todo su territorio: Francia, Italia, Portugal, Grecia, y Eslovenia. El resto de países de la Unión Europea tienen una presencia muy poco relevante (Austria y Finlandia), marginal o nula de los peajes directos ${ }^{20}$. En el caso de Austria (desde 2004) y Alemania (desde 2005) se han introducido recientemente peajes para los vehículos pesados en toda la red de autopistas. Tenemos pues, un sistema mixto de financiación de las autopistas muy peculiar en el contexto de la Unión Europea, que tiene un rasgo diferencial de carácter aún más excepcional: la gran variabilidad interterritorial del peso de los peajes. Estas características confieren una gran singularidad al modelo español de financiación de autopistas en el marco de la UE.

Dados los problemas que enfrenta de cara al futuro la política de carreteras en España, una reforma adecuada podría ser la transferencia de las competencias de gestión de las autopistas estatales a las Comunidades Autónomas. Este cambio comportaría la responsabilidad regional tanto por lo que respecta al mantenimiento y reposición, como las decisiones relativas a la financiación de estas funciones (bien con el presupuesto, bien con el pago por usuario -por peaje directo o mediante fórmulas alternativas-). Por una parte, no existen motivos para pensar que la eficiencia de los gobiernos regionales tenga que ser inferior a la del gobierno central en esta materia; más bien al contrario, dadas sus mayores restricciones financieras (que, por cierto, habrían hecho mucho menos probables concesiones como las que el Estado se propone rescatar a finales de 2010). Por otra parte, esto ayudaría a racionalizar el sistema irracional de financiación de las autopistas en España, pues el peaje mantiene unas disparidades territoriales muy importantes. De este modo, cada región podría decidir si recurre al pago por usuarios (mayoritariamente residentes en la propia región) o a las asignaciones del presupuesto para financiar el mantenimiento y reposición de las vías. Y esto facilitaría que el criterio escogido se pueda aplicar a todas las vías de gran capacidad en la región ${ }^{21}$.

\footnotetext{
${ }^{20}$ Polonia se encuentra en una situación intermedia, con un peso de los peajes próximo al 50 por 100. Información detallada sobre las características de financiación de las autopistas en Europa pueden encontrarse en ALBALATE, BEL y FAGEDA (2009).

${ }^{21}$ En principio, la homogeneización del sistema de financiación de autopistas en España podría ser también decidido por el gobierno central. Pero los antecedentes de la política de autopistas en los últimos 25 años hacen ingenuo pensar que esto pueda ocurrir en la práctica (BEL y FAGEDA, 2005).
} 
Este tipo de reforma no es del todo original. Es, por ejemplo, el sistema que existe en EEUU. La mayoría de autopistas estatales en EEUU fue planificada y construida por el gobierno federal, como parte del Sistema Interestatal de Autopistas, impulsado desde 1956. Más recientemente, las competencias sobre las autopistas interestatales fueron transferidas a los Estados, que están encargados de su gestión, y de la financiación de su mantenimiento y reposición. Cada Estado toma las decisiones que considera más pertinentes, y no es extraño, especialmente en la Costa Este, que una misma autopista tenga peajes en un Estado, no los tenga en el siguiente, pero vuelva a tener peajes en el de más allá. Al fin y al cabo, los residentes en cada Estado deciden si pagan peajes o pagan más impuestos (aunque se requiere el consentimiento de las autoridades federales para establecer peajes en autopistas libres que fueron construidas con fondos federales) ${ }^{22}$. Como es lógico, en la medida en que se internalizan beneficios y costes de sus decisiones, las divergencias entre Estados no provocan controversias como las que existen en España al respecto, ni causan distorsiones en la competencia por aplicar diferentes precios (con la gratuidad en muchas vías en el límite) para servicios similares.

La gestión regional de las «autopistas de larga distancia» no existe solo en EEUU. También existe en España, pues las provincias vascas tienen la competencia de gestión sobre casi todas las vías inter-autonómicas que discurren por la región.

Una nota sobre solidaridad territorial es conveniente antes de concluir: un sistema de este tipo puede ser perfectamente compatible con objetivos de reequilibrio territorial de los gobiernos para financiar obra nueva (que, en todo caso, han dejado de tener en este campo la relevancia que tuvieron varias décadas atrás), mediante mecanismos ya existentes, como el Fondo de Cooperación Interterritorial-FCI-. Al fin y al cabo, la región de Canarias (como la de Baleares) ya tiene competencia exclusiva sobre carreteras, y en el caso de Canarias se aplican fondos procedentes del FCI para financiar parte de sus actuaciones.

\section{Conclusiones}

Después de una década «maravillosa» por lo que respecta a la inversión en infraestructuras, en la que objetivos meta-políticos han dominado sobre las consideraciones propias de economía del transporte; debe ser urgente rescatar tres cuestiones respecto a cada proyecto de inversión: ¿cuánto cuesta?; ¿qué hace?; ¿qué se podría hacer alternativamente con los recursos implicados? El hecho de volver a considerar estas cuestiones ofrece una gran potencial de mejora de la política de infraestructuras.

\footnotetext{
${ }^{22}$ Efectivamente, sería recomendable que el gobierno central (o mejor una agencia reguladora independiente) retuviese potestades regulatorias sobre las autopistas inter-autonómicas para prevenir que, en aquellas vías en que exista un nivel de uso muy alto por no-residentes en la región, los peajes fuesen fijados por encima del coste para explotar a los no-residentes. Agradezco a Ángel de la Fuente esta sugerencia
} 
En este trabajo se han discutido algunas reformas de tipo organizativo e institucional que mejorarían los incentivos para una política más racional, que tenga más en cuenta el beneficio social potencial de las actuaciones de infraestructuras. Una primera reforma es instaurar un modelo de gestión individual en todas aquellas infraestructuras que sean un equipamiento singular, y carezcan de características de red. Este es el caso específico de los aeropuertos; también por los puertos, que ya han experimentado avances moderados en tal dirección. Esto forzaría a que los gobiernos territoriales se hagan responsables, o al menos corresponsables, de los efectos financieros de las decisiones relativas a las infraestructuras. El gobierno central, o mejor aún una agencia reguladora independiente de ámbito estatal, debería retener competencias regulatorias. Además, sería conveniente un mayor activismo y papel en este terreno de la Comisión Nacional de la Competencia.

La extensión de la red de AVE ha llegado ya hace tiempo a un nivel absurdo. Esto es de conocimiento cada vez más generalizado, pero nadie se atreve a «ponerle el cascabel a este gato». Sólo una reforma institucional, preferentemente de la UE, podría ayudar a racionalizar la política. Habida cuenta de que el ferrocarril de pasajeros a larga distancia no disfruta de la calificación formal de obligación de servicio público: (a) la UE debería evitar otorgar subvenciones a infraestructuras que no satisfacen obligaciones de servicio público, y (b) la Comisión Europea debería ser más beligerante contra las ayudas de explotación a las líneas ya existentes, de forma que la operación de la alta velocidad ferroviaria esté obligada a recuperar -al menoslos costes variables. En consecuencia con cambios de este tipo, una cuestión revestirá relevancia en el futuro: ¿deben mantenerse en operación las líneas que ni siquiera cubren costes variables, solo porque han sido construidas? Es fácil entender que las autoridades políticas quieran evitar situaciones ridículas como el abandono del servicio en líneas construidas a gran coste para un servicio muy exiguo. Pero el coste en términos de subvenciones de explotación para mantenerlas operativas impondrá fuertes perjuicios a los contribuyentes, además de distorsionar la competencia contra otros modos de transporte de viajeros.

Por lo que respecta a las autopistas, las inversiones en mantenimiento y reposición han adquirido prioridad sobre la creación de infraestructura nueva. Los avatares sufridos por las últimas concesiones de autopistas de peaje, que exigen voluminosas ayudas que pagarán los contribuyentes, constituyen una muestra fehaciente de esto. La combinación de una oferta política desde instancias gubernamentales con la demanda de grupos de interés privado cuyas inversiones disfrutan de una rentabilidad financiera garantizada está en el origen de decisiones desafortunadas en materia de concesiones de nuevas autopistas de peaje. Por ello, y dada la madurez alcanzada por la dotación de autopistas a nivel global de España, la trasferencia de las competencias de gestión a las regiones podría ayudar a superar el problema -ya clásico- que supone tener un modelo híbrido de financiación de las autopistas en España. Habida cuenta de la incapacidad mostrada por los órganos ejecutivos y legislativos centrales para resolver este problema, la regionalización permitiría que las regiones internalizaran los efectos de las decisiones tomadas sobre cómo financiar 
el mantenimiento y reposición de las infraestructuras viarias de gran capacidad. ¿Peaje directo, tasas de usuarios o financiación por presupuesto? Que cada región siga las preferencias de sus ciudadanos y que éstos soporten las consecuencias de las decisiones tomadas. Sin duda alguna, alinear adecuadamente la elección y la responsabilidad por las consecuencias de las elecciones efectuadas establecería incentivos indudables para racionalizar la política de infraestructuras también en este ámbito.

\section{Referencias bibliofgráficas}

[1] ADIF (2010): Memoria Económica. Ejercicio 2009. Madrid: Adif.

[2] ALBALATE, D. y BEL, G. (2011): «Cuando la economía no importa: Auge y esplendor de la Alta Velocidad en España», Revista de Economía Aplicada, 29 (55), en prensa.

[3] ALBALATE, D.; BEL, G. y FAGEDA, X. (2009): «Privatization and regulatory reform of toll motorways in Europe» Governance, 22 (2), 295-318.

[4] BEL, G. (1994): «Efectos imprevistos de la política de transporte», Revista de Econo mía Aplicada, 2 (6), 105-127.

[5] BEL, G. (1997): «Changes in travel time across modes and its impact on the demand for inter-urban rail travel», Transportation Research-E, 33 (1), 43-52.

[6] BEL, G. (2007): «Política de transporte: ¿Más recursos o mejor gestión?», Economis tas, 0 (111), 279-284.

[7] BEL, G. (2010): España, capital París. Origen y apoteosis del Estado radial. Barcelona: Destino.

[8] BEL, G. y FAGEDA, X. (2005): «Is a mixed funding model for the highway network sustainable over time? The Spanish case», Research in Transportation Economics, 15, 187-203.

[9] BEL, G. y FAGEDA, X. (2011): «La reforma del modelo de gestión de aeropuertos en España: ¿Gestión conjunta o individual?», Hacienda Pública Española/Revista de Eco nomía Pública, 196, en prensa.

[10] DE RUS, G. (2009): «La medición de la rentabilidad social de las infraestructuras de transporte», Investigaciones Regionales, 14, 187-210.

[11] DE RUS, G. e INGLADA, V. (1993): «Análisis coste-beneficio del tren de alta velocidad en España», Revista de Economía Aplicada, 1 (3), 27-48.

[12] DE RUS, G. e INGLADA, V. (1997): «Cost-benefit analysis of the high-speed train in Spain», Annals of Regional Science, 31(2), 175-188.

[13] DE RUS, G., y ROMÁN, C. (2006): «Análisis económico de la línea de alta velocidad Madrid-Barcelona», Revista de Economía Aplicada, 14 (42), 35-79.

[14] EUROPEAN COMMISSION, DG-TREN (2002): Study on competition between air ports and the application of state aid rules. Final report, Volumes I and II. Bruselas: European Commission.

[15] KAGESON, P. (2009): Environmental aspects of inter-city passenger transport. OECD-International Transport Forum, Discussion Paper N. 2009-28. 
[16] MARTÍN, J. C.; ROMÁN, C. y VOLTES-DORTA, A. (2009): «A stochastic frontier analysis to estimate the relative efficiency of Spanish airports», Journal of Productivi ty Analysis, 31 (3), 163-176.

[17] MILLS, G. (1995):«Airports users don't pay enough - and now here's privatisation», Economic Papers, 14 (1), 73-84.

[18] NOLAN, J.; RITCHIE, P. y ROWCROFT, J. (2005): «Small market air service and regional policy», Journal of Transport Economics and Policy, 39 (3), 363-378.

[19] ROBINSON, J. A. y TORVIK, R. (2005): «White elephants», Journal of Public Eco nomics, 89 (2-3), 197-210.

[20] ROBUSTÉ, F. (2010): Criteris per a la priorització de les inversions en infraestruc tures. Mimeo.

[21] WILLIAMS, G. y PAGLIARI, R. (2004): «A comparative analysis of the application and use of public service obligations in air transport within the EU», Transport Policy, 11 (1), 55-66. 